\title{
Quelques configurations relationnelles de la douleur*
}

\author{
Michael Houseman (CNRS)
}

\section{(in F. Hérituer De la violence II, Paris, Editions Odile Jacob, 1999, pp.77-112)}

Je me propose ici de considérer une série de phénomènes organisés autour de la souffrance : la douleur chronique, la torture politique, des rites d'initiation et divers types de mises à mort publiques. Mon objet n'est pas d'analyser ces phénomènes en détails, mais de présenter, à titre spéculatif, un cadre possible pour leur mise en relation. En d'autres termes, à partir de ces exemples je cherche à poser quelques jalons pour une réflexion anthropologique sur la douleur qui se démarque du point de vue habituellement adopté pour aborder cette question en ce qu'elle privilégie, non pas l'expérience de la douleur en elle-même, mais l'inscription de cette expérience dans un réseau particulier de relations.

Prenons comme point de départ l'article de Pierre Clastres (1973), "De la torture dans les sociétés primitives", dans lequel, à partir de données amérindiennes, il établit un rapprochement entre initiation et torture :

"D'une tribu à l'autre, d'une région à l'autre, les techniques, les moyens de cruauté diffèrent ; mais la fin reste la même : il faut faire souffrir l'initié. Nous avons nousmême décrit ailleurs l'initiation des jeunes gens guayaki, dont on laboure le dos sur toute la surface. [...] On pourrait à l'infini multiplier les exemples qui tous nous apprendraient une seule et même chose : dans les sociétés primitives, la torture est l'essence du rituel d'initiation." (1973:116-7)

Pour Clastres, cette souffrance obligatoire trouve son expression exemplaire dans les mutilations corporelles. La douleur initiatique interviendrait comme mise à l'épreuve du courage des novices mais aussi et surtout comme écriture dans la chair. "Le but de l'initiation en son moment tortionnaire, écrit-il, c'est de marquer le corps : dans le rituel initiatique, la société imprime sa marque sur le corps des jeunes gens." (ibid. 118) Et plus loin : " [...] les cicatrices dessinées sur le corps, c'est le texte inscrit de la loi primitive, c'est en ce sens une écriture sur le corps." (ibid. 120)

Ces propos soulèvent d'importants problèmes : concernant ce qui serait "écrit" sur le corps des candidats ${ }^{1}$, concernant l'usage même de notions telles que "texte", "écriture" ou "loi" dans des sociétés sans écriture, etc. Toutefois, les réactions assez vives qu'a suscitées cet article à l'époque portaient avant tout sur la comparaison, quelque peu provocatrice, entre initiation et torture. Rappelant certaines évidences passées sous silence par Clastres -- que les rites d'initiation sont bien plus que "des formes artificiellement exacerbées de la souffrance infligée à autrui" (Margarido et Panoff 1974:140) et, réciproquement, que "la torture n'est pas tant la souffrance que son utilisation" (Bernard et Fisher 1974:137) -- les critiques mettent en avant une série de traits qui opposeraient ces deux phénomènes. Tout d'abord, la torture ne fait pas intervenir un apprentissage, alors que l'initiation comporte la transmission d'un savoir essentiel. Ensuite, la torture opère, clandestinement et de façon illégitime, l'avilissement des individus, leur dégradation et destruction, tandis que l'initiation affirme, publiquement,

\footnotetext{
* Je tiens à remercier F. Héritier et les participants à son séminaire "De la violence" ainsi que M. Bekombo, P. Boyer, Ch. Malamoud, M.-J. Pineau-Jamous, C. Severi, E. Viveiros de Castro et plus particulièrement M. Moisseeff, qui ont bien voulu lire et commenter une première version de ce travail.

${ }^{1}$ Pour Clastres, soucieux de souligner combien les sociétés sans état s'inscrivent à l'encontre de formations sociales fondées sur la hiérarchie, cette "loi primitive, cruellement enseignée, est une interdiction d'inégalité dont chacun se souviendra. [...] La marque sur le corps, égale sur tous les corps, énonce : Tu n'auras pas le désir du pouvoir, tu n'auras pas le désir de soumission (1973:119-120).
} 
l'accession légitime des novices à un nouveau statut social prééminent. Enfin, la torture serait "le domaine par excellence de l'arbitraire individuel, voire du pur caprice de l'homme qui tient autrui à sa merci", tandis que les supplices initiatiques seraient "strictement codifiés dans leur déroulement" (Margarido et Panoff 1974:141).

Sans être fausses, ces oppositions laissent toutefois beaucoup de choses dans l'ombre. Plus précisément, elles s'appliquent mieux à certains épisodes qu'à d'autres. Elles conviennent notamment très bien aux séquences retenues par Clastres lui-même : épreuves publiques ou semi-publiques comportant, de façon typique, des mutilations corporelles et où la volonté des candidats est respectée. La douleur y devient source d'honneur : en surmontant sa souffrance, le novice démontre son courage et sa force virile. De pareilles scènes n'ont évidemment pas d'équivalent dans la torture ; le contraste est donc maximal.

Mais, pour d'autres séquences rituelles, l'écart entre initiation et torture est nettement moins marqué. La littérature ethnographique abonde en épisodes initiatiques où, loin du regard du public (non initié), les candidats, contraints à vivre dans des conditions matérielles pénibles et humiliantes, constamment insultés et régulièrement battus, maintenus dans une attitude de soumission et de peur, sont astreints à des épreuves d'un tout autre ordre. Dans le poro des Senufo de la Côte d'Ivoire par exemple (Zempleni 1993), il s'agit, entre autres, de cruels "travaux" champêtres, administrés par la promotion précédente. Les novices apprennent que "attacher le village (du bois sacré)" c'est devoir courir autour de ce petit village sous de violents jets d'eau et de gravillons, que "cultiver ses champs" c'est devoir boire une décoction émétique qui les fait vomir péniblement, etc. Dans le rite so des Beti du Sud-Cameroun (Houseman 1986), beaucoup de ces brimades consistent en des chasses ironiques : "capturer" tel animal c'est saisir aussi vigoureusement que possible une masse d'épines qui déchire cruellement les mains, "chasser les sangliers" c'est marcher à quatre pattes pendant des heures à travers la forêt poursuivi par les initiateurs, "se reposer" après une telle séance de "chasse" c'est se tasser dans un abri de très faible hauteur dont le toit est fait de lianes épineuses et dont le sol boueux est plein d'excréments, etc. Par ailleurs, les novices apprennent que "souffler dans une corne" (tout homme adulte possède une corne dans laquelle il souffle pour éloigner les mauvais esprits), c'est souffler dans l'anus des initiateurs, que "forger" c'est se faire écraser le doigt avec une bûche, que "se laver" c'est se plonger dans un marigot fortement pimenté et rempli d'excréments et d'autres saletés, que "cueillir des noix de cola" c'est devoir grimper à un arbre habité par des fourmis noires dont les morsures sont extrêmement douloureuses, etc.

Ces supplices que l'on fait subir dans les coulisses de la scène initiatique et pour lesquels l'improvisation des initiateurs est (jusqu'à un certain point) encouragée, sont clairement orientés vers l'avilissement et l'humiliation des novices. Les critères de discrimination entre torture et initiation proposés -- apprentissage, non-clandestinité, absence d'invention -- ont ici moins de prise. La douleur en question n'est pas signifiante et honorable, elle est dépourvue de sens et dégradante : les novices sont contraints -- ne serait-ce que par leur soumission -- d'affirmer, sous forme de paradoxes, des propos manifestement contradictoires ou absurdes : être chasseur c'est être chassé, être propre c'est être sale, être un homme c'est ne pas l'être, etc. Nous sommes ici très proches de la fameuse scène de torture de 1984 de George Orwell (1950), où Winston, relié à un générateur d'électricité, est péniblement amené à admettre que deux plus deux peut être égal à cinq ${ }^{2}$.

\footnotetext{
${ }^{2}$ Voici l'épisode en question (G. Orwell, 1984, Paris, Gallimard, 1950 : 360-3) :

"O'Brien présenta à Winston le dos de sa main gauche levée. Le pouce était caché, les quatre doigts étendus. "Combien est-ce que je vous montre de doigts, Winston ?" "Quatre." Le mot se termina par un halètement de douleur. L'aiguille du cadran était montée à cinquante-cinq. La sueur jaillie de son corps avait recouvert Winston tout entier. L'air lui déchirait les poumons et ressortait en gémissements
} 
Comme je l'ai annoncé, ce qui m'intéresse ici c'est d'analyser certaines des configurations relationnelles qui sous-tendent la souffrance. Le rapprochement que je serai amené à établir entre initiation et torture repose donc, non sur le degré d'identité qui peut exister entre ces deux phénomènes, mais sur les rapports qu'il y a lieu d'établir entre les phénomènes de douleur qui y interviennent. En d'autres termes, je suis à la recherche d'un critère de distinction qui permette non seulement de différencier torture et initiation, mais aussi d'inscrire cette différence dans un cadre plus large permettant de discriminer différents phénomènes où intervient de façon centrale la douleur. Un tel cadre, me semble-t-il, doit partir du truisme suivant : la différence entre douleur initiatique et douleur dans la torture tient avant tout au contexte relationnel qui, dans l'un et l'autre cas, préside à l'imposition de la souffrance. En bref, c'est à travers l'étude des configurations relationnelles de la douleur qu'il faut aborder ce problème.

Considérons tout d'abord, dans cette perspective, cette modalité particulièrement épurée de la souffrance qu'est la douleur chronique. En ce que celle-ci n'engage qu'une seule personne, elle représenterait une forme particulièrement simple ou élémentaire de l'expérience de la douleur.

\section{La douleur chronique}

Ce concept mal défini se réfère à une douleur importante et persistante, rebelle aux thérapeutiques, souvent d'étiologie incertaine et typiquement sans fondement organique évident : migraines, maux de dos ou d'articulations, gênes postopératoires, suites d'accidents, etc. Il existe une abondante littérature médicale sur la douleur chronique, notamment depuis la mise en place de centres et de services consacrés à son traitement. Les ethnologues (entre autres) qui travaillent depuis peu dans ces centres dans une optique autre que biomédicale (cf. par exemple Delvecchio-Good et al. 1992) se sont penchés sur les qualités perceptives, conceptuelles, etc. des "mondes" où vivent des patients souffrant de douleurs chroniques.

L'univers de la douleur chronique peut être caractérisé par trois traits principaux. Le premier est un dérèglement de la perception ordinaire du temps et de l'espace. Le temps peut se ralentir péniblement, ou au contraire s'accélérer à une trop grande vitesse. Assujettie à de tels effets de dilatation ou de contraction du temps, la personne souffrante appréhende

profonds qu'il ne pouvait arrêter, même en serrant les dents. O'Brien le surveillait, quatre doigts levés. Il ramena le levier en arrière. Cette fois, la souffrance ne s'apaisa que légèrement. "Combien de doigts, Winston?" "Quatre." L'aiguille monta à soixante. "Combien de doigts, Winston?" Quatre! Quatre! Que puis-je dire d'autre? Quatre."

L'aiguille avait dû monter encore, il ne la regardait pas. Le visage lourd et sévère et les quatre doigts emplissaient le champ de sa vision. Les doigts étaient dressés devant ses yeux comme des piliers énormes, indistincts, qui semblaient vibrer. Mais il y en avait indubitablement quatre. "Combien de doigts, Winston?" "Cinq! Cinq! Cinq!" "Non, Winston, c'est inutile. Vous mentez. Vous pensez encore qu'il y en a quatre. Combien de doigts, s'il vous plaît ?" "Quatre! Cinq! Quatre! Tout ce que vous voudrez. Mais arrêtez cela. Arrêtez cette douleur!"

[La supplice continue avec l'aiguille du cadran qui monte progressivement à soixante-dix, soixante-quinze...]

L'aiguille était peut-être à quatre-vingts, quatre-vingt-dix. Winston ne pouvait se rappeler que par intermittences pourquoi il souffrait. Derrière ses paupières serrées, une forêt de doigts semblaient sortir entrelacés, disparaître l'un derrière l'autre, réapparaître encore. Il essayait de les compter, il ne se souvenait pas pourquoi. Il savait seulement qu'il était impossible de les compter, à cause d'une mystérieuse identité entre quatre et cinq. La souffrance s'éteignit une fois de plus. Quand il ouvrit les yeux, ce fut pour constater qu'il voyait encore la même chose. D'innombrables doigts, comme des arbres mobiles, dévalaient à droite et à gauche, se croisant et se recroisant. Il referma les yeux. "Je montre combien de doigts, Winston?" "Je ne sais. Je ne sais. Vous me tuerez si vous faites encore cela. Quatre, cinq, six, en toute honnêteté, je ne sais pas." "Mieux", dit O'Brien." 
difficilement la durée comme un passage d'intervalles réguliers ; il devient compliqué de prévoir les événements à l'avance. Du côté de la perception de l'espace, interviennent tout d'abord des confusions entre l'intérieur et l'extérieur du corps. Dans l'état d'hypersensibilité auquel mène la douleur chronique, les frontières de l'individu deviennent floues : des stimuli externes (des bruits par exemple) peuvent être ressentis comme des intrusions violentes ou, au contraire, un malaise interne peut être projeté sur des éléments de l'environnement. On observe en même temps une confusion entre la partie et le tout du corps, une difficulté à localiser la douleur, ressentie à la fois comme associée à un certain endroit (là où j'ai mal) et vécue comme un fait total (je suis mal). Dans des dérèglements de ce genre, touchant à des repères fondamentaux de la personne, ce n'est pas seulement la perception mais l'appréhension du soi qui se trouve menacée (Good 1992:41; Bernard 1982). Un second trait du monde de la douleur chronique consiste en une coupure radicale entre celui qui souffre et les autres. Elaine Scarry (1985:4) résume la situation parfaitement : "La douleur arrive parmi nous sans pouvoir être partagée, à la fois ce qui ne peut être niée [par celui qui la ressent] et ce qui ne peut être confirmée [par celui qui ne la ressent pas]." De nombreux auteurs ont souligné l'inadéquation du langage face à la douleur, les effets d'alexithymie et les difficultés de communication qu'elle provoque (par exemple : Hilbert 1984; Violon 1992:67, et sur un mode littéraire Woolf 1981:15-16). Entouré de personnes qui, inévitablement, "ne peuvent pas comprendre", l'individu souffrant est renvoyé à lui-même. Enfin, le monde de la douleur chronique est animé par une urgence explicative, un besoin impératif d'identifier les causes et les raisons de la souffrance : "La douleur n'invite pas l'interprétation [...], elle semble l'exiger." (Morris 1991:34) Cette recherche ontologique devient d'autant plus pressante qu'elle est fatalement exacerbée par un questionnement de type existentiel : pourquoi moi ?

La conjugaison de ces trois caractéristiques -- dissolution des frontières habituelles du moi, retranchement sur soi et besoin pressant d'explication -- aboutit à ce qui représente peut-être la caractéristique première de l'expérience de la douleur chronique, à savoir une tendance vers le dédoublement. Celui-ci s'opère sur plusieurs registres recouvrant différentes dimensions (psychologique, physiologique, sociologique) : "entre le moi et son existence corporelle" (Buytendjik 1951:16), mais aussi entre le moi endolori et le moi sans douleur ("l'homme qui souffre a un autre corps et il est un autre homme" [Buytendjik 1978:783]), entre un moi inconscient dont les motivations m'échappent et le moi conscient condamné à en subir les peines, entre le moi privé et le moi qu'exige la compagnie d'autrui, etc. (Good 1992). Dans l'ensemble, ces divers mouvements de dédoublement, engagés afin de se situer par rapport à sa douleur, s'organisent autour de deux stratégies (Jackson 1994). L'une consiste en une objectivation du mal : la douleur serait une réalité physique qu'il convient de reconnaître en tant que telle pour pouvoir lui faire face ; il s'agit d'un dysfonctionnement du corps pour lequel il faut rechercher un traitement approprié ou, à défaut, qu'il faut apprendre à accepter. L'autre stratégie consiste en une subjectivisation de la souffrance : la douleur serait un phénomène en partie mental sur lequel le patient peut donc avoir une certaine emprise ; on estime pouvoir maîtriser le mal en changeant d'attitude ${ }^{1}$. Cependant, face à une forte douleur

\footnotetext{
${ }^{1}$ Ces deux voies stratégiques correspondent à ce que Baszanger (1995) identifie comme les deux modèles de la prise en charge médicale actuelle de la douleur chronique : celui de la "guérison-technique" et celui de la "guérison-gestion". Les divers centres de traitement de la douleur en France (et aux Etats-Unis, cf. Csordas et Clark 1992, cité dans Baszanger 1995) peuvent être situés sur le continuum que définissent ces deux pôles. Pour Baszanger, ce clivage résulte des moyens techniques et des dispositions institutionnelles qui ont présidé à la mise en pratique de la théorie de la douleur dite "de la porte" (gate control theory) introduite en 1965 par Melzack et Wall (Melzack et Wall 1983) :

"[...]en posant l'existence d'une barrière modulable dans le système de transmission somatique de la douleur, la théorie de la porte dessine deux voies possibles pour obtenir une modulation dans le sens de la réduction du message douloureux, voies qui ne sont pas exclusives l'une de l'autre, et c'est d'ailleurs le
} 
qui dure, ni l'une ni l'autre de ces voies ne s'avère véritablement adéquate, car l'individu n'arrive ni à se séparer suffisamment de sa douleur, ni à la contrôler suffisamment. Il en résulte un mouvement de va-et-vient constant et quelque peu désespéré entre ces diverses alternatives : une quête de sens à jamais inassouvie.

A écouter les témoignages de personnes souffrant de douleurs chroniques, on ne peut qu'être impressionné par l'énergie qu'elles investissent dans cette tentative de comprendre leur état, de cerner les causes et les raisons de leurs peines, de préciser la nature de cette relation maléfique qui les accable : celle entre moi et ce "X" qui est à l'origine de la souffrance. Ainsi il arrive souvent que la douleur elle-même se trouve dotée d'intentionnalités, devenant sinon un homuncule tout du moins un interlocuteur éventuel -- un "elle" ou "ça" -- auquel l'individu se soumet ou non, avec lequel il lutte ou négocie, etc. De ce point de vue, on ne doit pas s'étonner de découvrir qu'un aspect essentiel de la quasi-totalité des traitements non biomédicaux de la souffrance consiste en l'identification de cet autre, c'est-à-dire du facteur causal ou plutôt de l'agent responsable dont l'existence semble présupposée par l'expérience même de la douleur chronique : dieux, ancêtres, esprits, sorciers, etc. Dans des centres de traitement de la douleur où une telle démarche est largement exclue, il est toutefois remarquable de constater à quel point le réseau de relations autour du patient est spontanément investi de spéculations de ce genre. Ainsi, ce sont les membres de la famille, le personnel soignant, d'autres malades, etc., qui peuvent devenir, aux yeux des patients, autant d'agents contribuant à leur malheur (Jackson 1994).

L'individu soumis à l'emprise d'une douleur chronique cherche continuellement à instaurer une séparation ou division au sein de sa propre personne. Il tente vainement d'établir une relation duelle stable là où elle ne saurait subsister. De ce point de vue, la douleur chronique est caractérisée par une configuration relationnelle qui est à la fois plus qu'une simple unité et moins qu'une authentique dualité. Qualifions-la de configuration de "un et demi". Une analogie avec la neurophysiologie de la vision (que je simplifie ici évidemment à outrance) aidera à mieux comprendre cette idée. La vision binoculaire permet une perception à trois dimensions. En revanche, la vision monoculaire ne permet qu'une perception à deux dimensions, sans profondeur de champ. Or, au niveau des représentations mentales, on distingue également un palier intermédiaire où interviennent, de façon quasi automatique, des mécanismes perceptifs enclenchés par une série de repères indicatifs d'une troisième dimension : la superposition partielle de certains objets, les gradients de luminosité, les angles d'intersection des bords, etc. Les "esquisses à deux dimensions et demi" (Marr 1982) qui en résultent sont bien plus que de simples représentations à plat. Toutefois, elles ne sont pas non plus véritablement tridimensionnelles : les mécanismes concernés aboutissent aisément à des incohérences, comme en témoignent de nombreuses illusions d'optique (flèches de Muller, cube de Necker). La douleur chronique, marquée par un dédoublement inachevé et intrinsèquement instable, occupe sur le plan de son schéma interactif caractéristique une place analogue à celle qu'occupent ces esquisses visuelles intermédiaires.

Le caractère élémentaire de la douleur chronique n'exclut donc pas qu'elle soit complexe. Ainsi, malgré l'absence de tout protagoniste réel, la personne souffrante œuvre à situer sa douleur dans un cadre interactif, attribuant par exemple à celle-ci des intentionnalités là où il ne peut y en avoir. Dans ces conditions, ne faut-il pas reconnaître dans la supposition d'une altérité agissante un aspect essentiel de l'expérience douloureuse ? En d'autres termes,

grand apport de la théorie que de les avoir présentées dans un seul modèle. La première voie passe par une action sur les structures organiques ou des médiateurs chimiques (des nœuds, des ganglions, la moelle épinière, des synapses, le thalamus, des enzymes...), tandis que l'autre s'adresse par le biais de contrôles descendants à des processus mentaux (les processus cognitifs par exemple)" (Baszanger 1995:266-7). 
d'un point de vue analytique, le prototype de la douleur serait une douleur infligée. Dans le cas de la douleur perçue comme infligée, l'indétermination ontologique quant à l'origine de la souffrance est clairement levée à au moins un niveau : j'ai mal parce que quelqu'un me fait mal. La souffrance expressément imposée à un autre peut être appréhendée en termes d'intentionnalités imputables à des agents externes. Même si les motivations et les mécanismes en jeu ne sont pas clairement définis, leur existence ne fait l'objet d'aucun doute. Une telle perspective impose un certain recentrage dans l'interprétation des phénomènes de douleur. Jusqu'ici, l'étude de la douleur a été dominée par une réflexion médicale (et philosophique) qui met l'accent sur la douleur ressentie, c'est-à-dire en tant que modalité particulière de l'expérience corporelle. Je propose de renverser cette conception en prenant comme cas paradigmatique de la douleur, non pas la douleur simplement ressentie, mais la douleur infligée. Dans cette optique, il conviendrait d'envisager la douleur chronique comme une souffrance infligée dont les intentionnalités et les agents concernés seraient particulièrement difficiles à identifier.

Des douleurs plus passagères et moins importantes, comme lorsque je cogne malencontreusement mon orteil contre un caillou, ne sont pas nécessairement vécues comme infligées. Ne provoquant pas la supposition d'une instance agissante, elles correspondraient à des cas de douleur fondée sur une configuration relationnelle de type "un" (sans le "demi" en plus). Une représentation de la souffrance comme animée par une intentionnalité dépendrait donc de l'intensité, de la durée et, éventuellement, de la répétition de la douleur concernée. Toutefois, dans l'optique adoptée ici, l'attribution du mal à une altérité supposée représente une virtualité de toute douleur. Combien d'entre nous ne voient-ils pas sur le coup dans ce malheureux caillou (ou dans son pied, ou dans sa propre personne) un antagoniste virtuel, auquel nous adressons éventuellement des paroles de colère, de reproche ou de défi ?

Reconnaître ainsi une préséance analytique à la douleur infligée a l'avantage d'impliquer une simplification considérable de l'objet. Dans le cadre de la douleur infligée, les deux distinctions qui hantent toutes les discussions modernes sur la douleur deviennent plus ou moins pertinentes. Il s'agit d'un côté de l'opposition entre "douleur", sensation nocive immédiate et localisable (un stimulus nociceptif), et "souffrance" en tant qu'état de détresse plus diffuse, consciemment supporté, et de l'autre, entre "douleur physique" et "douleur psychique" ou "morale" (cf. par exemple Rey 1993). Dans la mesure où la douleur infligée est tenue pour inséparable des conditions relationnelles au travers desquelles elle se réalise, elle comporte nécessairement une part de réflexivité et donc de "souffrance". De façon réciproque, l'intensité effective des stimuli nociceptifs peut être d'une importance secondaire ; l'imposition volontaire par autrui de la peur, de la gêne ou d'autres expériences corporelles fondées sur des phénomènes psychologiques peut également être ressentie comme douloureuse. Par ailleurs, et il s'agit d'une autre simplification, des questions d'ontologie (quoi?) et des interrogations existentielles (pourquoi moi?) deviennent, dans le cadre de la douleur infligée, convergeantes, tendant à trouver une même réponse dans l'identité de l'agent responsable (qui?). En même temps, cette façon de voir les choses entraîne un certain épaississement de l'objet : dans la mesure où la douleur infligée demeure indissociable de son contexte interactif, elle n'est jamais qu'un phénomène nociceptif. Or, cela représente incontestablement pour l'ethnologue un avantage : une prise en compte systématique de ces contextes permet d'ouvrir une voie nouvelle dans l'étude comparative des expériences de la douleur. C'est cette voie que je me propose de poursuivre maintenant.

Afin de dépasser une configuration relationnelle de "un et demi" caractéristique de la douleur chronique, singulière, il est nécessaire d'envisager le cas où celui qui inflige la douleur est indéniablement présent. $\mathrm{La}$ torture politique en fournit un exemple particulièrement saillant. 


\section{La torture politique ${ }^{2}$}

J'ai déjà signalé certains éléments qui permettent de différencier torture et supplices initiatiques. La torture, dont le but est essentiellement d'anéantir le supplicié, comporterait uniquement une violence destructrice et déshonorante, tandis que l'initiation, qui vise avant tout à la transformation du novice, incorporerait une violence destructrice, mais aussi une souffrance constructive ou honorable. Cette différence de nature s'exprime également sur le plan de l'intensité, de la durée et surtout du rythme des peines imposées. Certaines épreuves initiatiques peuvent être extrêmement douloureuses et, dans certains cas, la torture peut se limiter à des violences d'ordre psychique. Toutefois, dans les rites d'initiation la douleur intervient de façon ponctuelle et s'arrête à la limite du supportable, tandis que dans la torture, par définition, elle s'accroît en intensité pour dépasser ce seuil. Dans un cas, les morts sont exceptionnelles, dans l'autre, elles sont monnaie courante.

Il est toutefois étonnant de constater la similarité des techniques d'intimidation et de dégradation régulièrement employées dans l'initiation et dans la torture : menaces, insultes, privation de sommeil, de vêtements, de nourriture, etc., passages à tabac, maintien de positions corporelles inconfortables, exposition au froid ou au chaud, etc. Plus encore, de nombreuses pratiques de torture ont, comme les brimades initiatiques (cf. supra), un caractère cruellement ironique. Ainsi, dans des lieux de détention, parfois désignés de façon sardonique ("la maison du rire" à Dubai [Forrest 1996:112], les "chambres d'amis" en Grèce [Scarry 1985:23]), ont lieu des opérations dans lesquelles des objets domestiques ordinaires -chaises, cadres de lit, baignoires, cuisinières, pilons, bâtons à écraser des épices ou des grains, appareils ménagers, stylos, etc. -- sont violemment retournés contre leurs utilisateurs. L'évocation d'objets et d'activités familières, notamment au travers de "petits noms" que les tortionnaires utilisent partout pour désigner ironiquement les punitions qu'ils administrent, est abondamment attestée (Forrest 1996; Jempson 1996) : le téléphone, l'hélicoptère, le phonographe, le poulet rôti, l'avion, la cloche, boire un soda, le sandwich, faire le planton, faire de la moto, etc. Nombreux sont les récits (par exemple Alleg 1961) qui permettent d'apprécier in situ ce mélange de badinage et de cruauté si caractéristique à la fois des brimades initiatiques et des activités de torture.

Il y a cependant une "technique" utilisée à la fois dans l'initiation et dans la torture qui fait ressortir une différence radicale entre ces deux phénomènes : la mise à mort simulée. Les menaces de mort et les fausses exécutions sont un aspect on ne peut plus courant de la torture. De même, une prétendue mise à mort des novices est un phénomène que l'on retrouve constamment dans les initiations masculines.

$\mathrm{Au}$ cours des rituels d'initiation, les jeunes sont, de façon caractéristique, arrachés de force au village où retentissent les hurlements et les pleurs des non-initiés, c'est-à-dire des femmes et des enfants ; ou encore, les non-initiés assistent de loin à des mises en scène du meurtre des novices. Ces séquences comportent suffisamment de violences, tant sur le plan discursif que sur celui des actions -- les cris et les lamentations des non-initiés, les menaces sans appel des initiateurs, les souffrances réellement subies par les novices, etc. -- pour que l'ensemble des participants puisse douter du caractère entièrement factice des opérations. D'ailleurs, le fait que de temps en temps il y ait des morts (accidentelles) et qu'il existe des procédures détaillées et connues de tous en prévision d'une telle éventualité témoignent du danger véritable de ces séquences. De ce point de vue, la peur et la tristesse qu'affichent les

\footnotetext{
${ }^{2}$ Cette section concerne en premier lieu la torture exercée au service d'une autorité ou d'une idéologie politique, c'est-à-dire hors du cadre de la jurisprudence, telle qu'elle s'est généralisée au cours du XXème siècle (pour une récente histoire de la torture, cf. Peters 1985).
} 
femmes et enfants exclus, l'angoisse des novices et l'acharnement cruel des initiateurs ne relèvent, ni pour les uns ni pour les autres, d'un simple jeu inoffensif. En même temps, la mort initiatique comporte une double collusion, à la fois (notamment dans sa préparation) entre les initiateurs et les non-initiés, et (généralement après coup) entre les initiateurs et les novices eux-mêmes. D'un côté, les novices sont rendus complices des initiateurs vis-à-vis des non-initiés : le caractère simulé de leur mort devient, par la suite, une révélation qu'ils doivent cacher aux femmes et aux enfants. De l'autre, les non-initiés eux-mêmes (qui n'ignorent pas que les candidats ne sont pas réellement tués) sont rendus complices des initiateurs vis-à-vis des novices : en feignant, eux aussi, la mise à mort des candidats, ils concourent à l'initiation de ces derniers et, par là, à leur accession au statut d'adulte. Il est évident que ces deux attitudes, celle des novices et celle des non-initiés, se répondent : c'est parce que les candidats collaborent (parfois contre leur gré) à la simulation de leur mise à mort, que les non-initiés peuvent faire de même, et c'est parce que ceux-ci agissent ainsi que la simulation à laquelle participent les candidats peut (par la suite) se constituer en un "secret" qui ne doit pas leur être révélé. De façon générale, la douleur initiatique se situe dans cette dynamique de dissimulation complexe définie par une articulation interdépendante d'au moins trois points de vue : celui des novices, celui des non-initiés et celui des initiateurs (Houseman 1993).

Dans le cadre de la torture, des mises à mort simulées auxquelles participent à la fois tortionnaires, victime et personnes extérieures telles que la famille ou les amis de la victime, sont exceptionnelles. On a à faire soit à des exécutions factices ayant lieu dans le huis clos entre tortionnaires et victimes, soit à des mises à mort publiques ou semi-publiques, mais alors authentiques (cf. par exemple les cas rapportés dans Jempson 1996:81-86). Le premier type de mise en scène, celui des fausses exécutions, fait partie du processus d'interrogation par lequel le tortionnaire tente de tirer des renseignements du prisonnier : il s'agit d'un moyen supplémentaire pour "casser" la victime. Le second type de mise en scène, celui des véritables exécutions plus ou moins publiques, vise avant tout l'intimidation de personnes autres que la victime : les membres de sa famille, ses voisins, ses camarades, etc. Il relève de ce que Shue (1978) appelle l'aspect "terrorisant" (terroristic en anglais) de la torture, orienté vers l'extérieur, vers ceux qu'on peut qualifier de "destinataires" de la douleur du supplicié (tout comme les non-initiés seraient les "destinataires" du secret que livrent douloureusement les initiateurs aux novices [Zempleni 1976], à savoir le caractère simulé de leur mise à mort). On retrouve dans ces deux types de mise en scène les deux dimensions relationnelles qui, de façon générale, président à la souffrance dans la torture politique : d'un côté celle de l'interrogation, centrée sur la relation entre le tortionnaire et sa victime, de l'autre celle de la terreur, centrée sur la relation entre le tortionnaire et des tierces personnes. Mais regardons ces deux relations de plus près. La relation d'interrogation entre le tortionnaire et sa victime

\footnotetext{
${ }^{3}$ En l'absence d'une description détaillée, il est difficile de restituer la subtilité de cette dynamique triangulaire. On la retrouve sous une forme condensée par exemple dans un des traits récurrents des initiations masculines où les non-initiés, installés hors de vue des novices et des initiateurs, restent néanmoins en communication auditive avec eux (Cohen 1964). Ainsi, dans le rite so des Beti du Cameroun, au moment de "la mort du so", les femmes et les enfants, installés à l'écart, doivent crier pour ne pas entendre les cris des candidats qui "meurent", lesquels entendent donc forcément les femmes (Houseman 1993). Une scène tirée du rite tsoo des Wagania du Zaire (Droogers 1981), capte bien la complexité de ce type d'interaction où se conjuguent plusieurs niveaux de dissimulation. Lorsqu'il commence à pleuvoir, les hommes adultes quittent l'enclos initiatique situé à l'extérieur du village, car les femmes et les autres non-initiés ne doivent pas savoir que les novices y vivent dans des maisons ; on leur explique qu'ils y demeurent sans toits et que c'est un grand oiseau, Mokumo, qui vient les protéger en cas de pluie. Ainsi, lorsqu'il pleut, les femmes viennent chanter et crier devant la palissade de l'enclos par sympathie pour les novices exposés à l'orage. En réponse aux chants des femmes, les novices doivent alors sortir sous la pluie dans la cours de l'enclos pour y jouer l'instrument qui est censé simuler le bruit de l'oiseau Mokumo venu pour les protéger, et dont le son est censé faire fuir les femmes. C'est pendant que les candidats sont amenés à ainsi tromper les non-initiés qu'un initiateur leur frappe les jambes avec un bâton.
} 
n'a de sens que dans la mesure où elle présuppose qu'il existe un lien entre la victime et des personnes extérieures. Sinon, pourquoi questionner la victime, et pourquoi celle-ci résisteraitelle à dévoiler des informations ? Il en est de même en ce qui concerne la relation de terreur entre le tortionnaire et les personnes extérieures : s'il n'existait pas de lien entre celles-ci et le supplicié, sur quoi se fonderait l'effet d'intimidation ? Ainsi, ce qui associe les deux dimensions relationnelles, interrogatrice et terrorisante, de la torture politique, et ce faisant, fonde l'unicité de ce phénomène, c'est que l'une et l'autre présupposent la présence d'une troisième relation, celle entre la victime et des tiers. Or, et c'est là un point essentiel, que les participants supposent que cette relation existe n'exige en rien que cette relation soit clairement définie, ni même qu'elle existe réellement. C'est là une différence fondamentale avec l'initiation où les diverses relations en jeu sont réalisées à travers la participation effective des uns et des autres dans le rituel. Une comparaison formelle de ces deux institutions permettra de faire mieux ressortir les implications de cette différence.

Dans la torture comme dans l'initiation, la douleur infligée se situe dans un contexte relationnel composé de trois termes : au triplet initiateurs/novices/non-initiés correspond l'ensemble tortionnaire/victime/personnes tierces. De plus, dans l'un et l'autre cas, on peut reconnaître à cette souffrance infligée deux fonctions ou orientations analogues. La violence qu'exercent les initiateurs envers les novices intervient à la fois pour détacher les novices des non-initiés avec qui ils étaient jusqu'alors identifiés, et pour intimider et subordonner les noninitiés. De façon similaire, la violence que le tortionnaire impose au prisonnier vise, du côté de l'interrogation, à séparer la victime de ses proches (qu'il est amené à trahir), et à semer la peur parmi des personnes extérieures. Toutefois, dans l'initiation, les relations reliant les trois termes en présence (initiateurs, novices et non-initiés) sont actualisées au cours du rite. C'est en effet cette actualisation qui les intègrent en un système unitaire : comme on l'a vu, le détachement des novices des non-initiés se fait avec la complicité des non-initiés, et inversement, l'intimidation des non-initiés se fait avec le concours des novices eux-mêmes. L'interdépendance nécessaire de ces diverses relations sur le plan de l'action impose ainsi à la démarche initiatique une forme bien particulière. Une initiation qui ne comporterait ni le concours des non-initiés dans les épreuves imposées aux novices, ni la complicité des novices dans l'intimidation des non-initiés, perdrait son caractère initiatique. En revanche, la pratique de la torture ne comporte pas obligatoirement une actualisation, et par là une détermination précise du lien entre la victime et des personnes tierces. Elle n'exige qu'une supposition de ce lien qui, n'étant pas contraint par des modalités de participation particulières, peut donc recevoir de la part des participants des interprétations extrêmement variables. C'est cette indétermination intrinsèque à la torture politique qui sous-tend les "dérapages" qui lui sont si familiers : interrogations poursuivies sans recherche véritable d'informations ${ }^{3}$ ou pratiques de terreur exercées sans discrimination. Plus encore, dans la mesure où le lien entre la victime et des personnes tierces peut être simplement supposé, les relations que ce lien est censé relier, à savoir la relation entre le tortionnaire et sa victime d'une part et la relation entre le tortionnaire et des personnes extérieures d'autre part, peuvent évoluer de façon plus ou moins indépendante. Ainsi, au cours de l'interrogation, l'intensité de la douleur infligée au prisonnier variera essentiellement en fonction des réponses de ce dernier ; les rapports entre le tortionnaire et les personnes tierces n'y jouent, en principe, aucun rôle. De façon réciproque, dans l'optique de la torture terrorisante, "le degré de souffrance imposé aux victimes est

\footnotetext{
${ }^{3}$ Comme le souligne Scarry (1985), cette quête d'information tend fréquemment sinon inévitablement, à devenir un prétexte dans le processus d'anéantissement du prisonnier. Cette transformation est d'autant plus facile que l'interrogation sous la torture correspond à une situation où toute négociation est largement illusoire : les moyens dont dispose le supplicié pour cacher ce qu'il peut savoir (à propos de ses proches par exemple) et les moyens dont il dispose pour démontrer qu'il ne sait rien, ne peuvent pas être nettement distingués.
} 
proportionnel non pas aux réponses de la victime, mais au retentissement attendu de la nouvelle de la torture sur d'autres personnes sur lesquelles la victime n'a pas de contrôle. [...] La victime est simplement un site sur lequel énormément de douleur est appliquée pour que d'autres en prennent connaissance et soient effrayés par cette éventualité" (Shue 1978:132) ${ }^{4}$. Il n'est évidemment nullement exclus qu'interrogation et terreur puissent dans certains cas se rejoindre : une même situation de torture peut être à la fois un moyen d'extraire des informations et un instrument de répression.

Dans l'initiation, la douleur infligée est inscrite dans un système où au moins trois termes sont intégrés en une configuration globale spécifique par trois relations agies. En revanche, les trois termes qui sous-tendent l'imposition de la douleur dans la torture (le tortionnaire, la victime et les proches de la victime) sont reliés par deux relations duelles -celle entre le tortionnaire et la victime et celle entre le tortionnaire et des personnes tierces -et la présupposition d'une troisième relation : celle entre la victime et des personnes tierces. On y observe donc un va-et-vient entre ces deux relations duelles, respectivement d'interrogation et de terreur ou, éventuellement, leur juxtaposition, mais très rarement, sinon jamais, une véritable triangulation systématique comme dans le cas de l'initiation. Dans cette optique, la configuration relationnelle présidant à la douleur infligée dans la torture serait plus qu'une simple dualité mais moins qu'une structure véritablement tridimensionnelle. En bref, elle serait une configuration de type "deux et demi". Ici, ce "demi" en plus consiste non pas, comme dans la douleur chronique, en la supposition d'une relation entre la personne souffrante et un protagoniste, mais en la supposition d'une relation (celle entre la victime et personnes tierces) entre deux relations (celle entre le tortionnaire et sa victime d'un côté et celle entre le tortionnaire et des personnes extérieures de l'autre).

Le prochain palier dans la progression des configurations relationnelles de la douleur que j'essaye de mettre en évidence ici nous renvoie à des situations où la douleur est publiquement infligée et où les personnes extérieures, les témoins ou "destinataires" de la souffrance, sont, à l'instar de l'initiation, partie prenante. C'est ce que nous trouvons dans l'usage proprement rituel de la douleur, dont j'analyserai deux autres exemples.

\section{L'usage rituel de la douleur : deux exemples}

Michel Foucault ouvre son livre Surveiller et punir (1975) avec une description de l'exécution publique de Robert-François Damiens (1757) à la suite de son attentat manqué contre Louis XV. Ce "spectacle de la souffrance" (Spierenburg 1984) aurait été, en effet, une des dernières grandes manifestations d'une période de plusieurs siècles caractérisée par une mise en scène publique et fastueuse, hautement ritualisée, de la souffrance corporelle ${ }^{4}$. En voici les éléments essentiels :

Une fois que Damiens est enchaîné à la table d'exécution dressée devant la foule assemblée, sa main droite, à laquelle on a préalablement attaché l'arme du crime, est sévèrement brûlée avec du soufre. Puis il est tenaillé aux bras, aux cuisses et à la poitrine avec des pinces chauffées au rouge ; un mélange ardent fait de plomb, d'huile, de poix et de résine fondue est versé dans les blessures ainsi pratiquées. Ses quatre

\footnotetext{
${ }^{4}$ D'ailleurs, continue Shue, il n'y a pas de raison en soi pour que la torture terrorisante ne s'achève pas en meurtre, "dans la mesure où on peut s'attendre à ce que les amis ou la famille de la victime propage la nouvelle concernant le prix à payer pour un comportement incompatible avec la loyauté" (1978 : 132-133).

${ }^{4} \mathrm{La}$ fréquence de tels spectacles varie avec le temps et d'un endroit à un autre. Par exemple, "les gens d'Arras, assistent à au moins une exécution capitale chaque mois pendant tout le XVIème siècle (Muchembled 1992:117), tandis que de 1651 à 1750, les habitants d'Amsterdam pouvaient assister à environ quatre exécutions par an, et tous les trois ans, au spectacle d'un criminel cassé sur la roue (Spierenburg 1984, Appendice B).
} 
membres sont alors reliés par des cordes à des chevaux. Il est ensuite écartelé, non sans difficulté, car après vingt-huit minutes, les juges demandent au bourreau de lui sectionner les articulations à l'aide d'un bistouri. La mort ne surviendra que lorsque le dernier de ses membres, le bras droit, fut séparé du tronc. Le supplice aura duré plus d'une heure (Rétat et al. 1979:358-60).

Les historiens invitent à analyser l'apparition de tels usages à la lumière d'évolutions que je vais rapidement évoquer. Tout d'abord, l'époque concernée est marquée par une transformation radicale des procédures judiciaires, c'est-à-dire le passage d'un système "accusatoire" régi par des ordalies et des duels, où la vérité se donne à voir directement dans le corps même des protagonistes, à un système "inquisitoire", où la vérité, qui se rapporte à des paroles qu'on extrait en secret, ne se réalise qu'au travers de l'énonciation d'un jugement (Spierenburg 1984; cf. aussi Asad 1993). Le corps supplicié du condamné devient alors "le support public d'une procédure qui était restée jusque-là dans l'ombre ; en lui, sur lui, l'acte de la justice doit devenir lisible pour tous" (Foucault 1975:53). Par ailleurs, cette période, "le temps des supplices" (Muchembled 1992), a connu un important développement urbain ainsi qu'une forte progression de la pauvreté dans les villes. Le recours à des exécutions spectaculaires aurait traduit une volonté à la fois de réprimer avec force les désordres internes à la ville et, par l'exposition des restes des corps des suppliciés à ses portes, de décourager l'entrée d'éventuels éléments nuisibles (cf. Muchembled 1992; Gonthier 1992). Enfin, cette époque est caractérisée par une centralisation accrue du pouvoir : "L'éclat des supplices et leurs suites expriment une brusque montée en puissance de l'État royal." (Muchembled 1992:121) Ce dernier, devenu de plus en plus sacralisé, s'attache "à prendre un monopole punitif qui ne lui appartenait pas complètement jusque-là" (ibid. 115). Dans cette optique, "la cérémonie du supplice fait éclater en plein jour le rapport de force qui donne son pouvoir à la loi" (Foucault 1975:61) ; elle représente un "opérateur politique" (ibid. 65), dans lequel l'intégrité du pouvoir momentanément blessé est restaurée de façon spectaculaire au travers de la destruction brutale du corps du condamné. Le but de cette cérémonie "est moins de rétablir un équilibre que de faire jouer, jusqu'à son point extrême, la dissymétrie entre le sujet qui a osé violer la loi, et le souverain tout-puissant qui fait valoir sa force" (ibid. 59-60).

Afin de dépasser cette idée du supplice public comme mode d'intimidation pour saisir sa spécificité en tant qu'acte rituel, il convient de centrer l'attention sur la masse des spectateurs, ceux que Foucault appelle "le personnage principal [des supplices] dont la présence réelle et immédiate est requise pour leur accomplissement" (ibid. 69). En effet, comme le remarque Cohen (1990:293), "les cérémonies d'exécution étaient [...] des rituels extrêmement riches et complexes dressant une relation à trois termes entre les autorités, le public et le condamné". Examinons donc de plus près la dynamique relationnelle unissant ces trois termes.

La douleur extrême imposée aux suppliciés marque de façon dramatique et immédiatement tangible une distance entre les autorités qui l'infligent et le condamné qui la subit. Mais en même temps, ce rapport d'altérité radicale est comme complété par une série d'identifications unissant le public à ces deux termes ainsi posés comme antinomiques. Les intenses souffrances qu'endure le condamné, et que les spectateurs peuvent s'estimer heureux de ne pas subir, amènent ceux-ci à s'éloigner de lui pour se ranger, en tant que bons citoyens ou sujets obéissants, du côté des autorités au nom desquelles le supplice s'accomplit. Mais simultanément, le caractère démesuré du châtiment qu'infligent ces autorités, sanction dont les spectateurs, en tant que sujets/citoyens, peuvent eux-mêmes devenir l'objet, conduit le public à se distancier des autorités pour se placer du côté du condamné. C'est la douleur infligée qui est en elle-même le "moteur" de ce réseau de relations intégrant des rapports de différentiation et d'identification contradictoires. Plus la souffrance qu'infligent les autorités au condamné est 
intense, plus l'éclat du pouvoir qu'elles représentent est grand, et plus l'écart qui les sépare du condamné devient important. Mais en même temps, plus le supplice est féroce, plus le public, à la fois se dissocie du condamné qui le subit pour se rapprocher des autorités et se dissocie des autorités qui l'infligent pour se rapprocher du condamné. Ainsi se met en place une relation rituelle complexe vis-à-vis du pouvoir, dans laquelle le sujet ou citoyen n'est assimilé ni à celui qui subit les supplices, ni à ceux qui les infligent mais, par ricochet, aux deux à la fois. Le corrélat affectif de cette position paradoxale, l'expression des sentiments qu'elle comporte, serait la fameuse "émotion d'échafaud" signalée non sans étonnement par divers auteurs (par exemple Bee 1983) : un mélange tout particulier de fascination horrifiée et de débridement confus.

Cette façon de voir les choses permet aussi d'éclairer quelque peu le statut ambivalent du bourreau. Celui-ci est à la fois un personnage très respecté, doté de pouvoirs bénéfiques, notamment de guérison, et un individu redouté comme source d'infamie : on évite de recevoir quelque chose directement de la main des bourreaux, lesquels sont contraints de vivre à la périphérie de la ville ou dans les bas quartiers, à communier à l'église en dernier, etc. (Delarue 1979; Spierenburg 1984:13-33). ${ }^{5}$ Nous avons déjà remarqué qu'au cours des supplices, les spectateurs sont virtuellement identifiés à la fois aux autorités et à la victime. Cependant, leur association à ces deux pôles de la relation de douleur infligée est définie de façon négative, indirectement et en fonction du réseau de relations établi au cours du rituel. En revanche, le bourreau est directement identifié à ces deux pôles et de façon positive, en raison d'un rapport de contiguïté qui est constitutif de son personnage. Ainsi, d'un côté, il est le représentant exécutif du pouvoir dont il porte l'uniforme et duquel il reçoit une rémunération (l'ambivalence vis-à-vis du bourreau ne fait son apparition que lorsque son poste est officiellement rétribué, évolution marquée par la mise en place de la procédure inquisitoire [Spierenburg 1984:25-26]). De l'autre, le bourreau, qui est souvent un ancien condamné, s'engage (comme le montrent divers témoignages) dans un terrible corps-à-corps intime avec la victime, dont la dépouille lui est souvent remise pour qu'il en dispose à sa guise. A la fois émanation des autorités et confondu avec les condamnés, le bourreau se présente donc comme un "double" en positif du public. Il intervient face à lui comme une sorte de méta-miroir réifiant : il renvoie aux spectateurs non pas une image de ce qu'ils sont, mais une incarnation des conditions relationnelles de ce que l'événement les fait devenir le temps de leur participation. Ce rapport horizontal entre le bourreau et le public peut désormais devenir, du point de vue de l'assistance, le pendant légitimant du rapport vertical entre les autorités et la victime. Ainsi, lorsqu'il arrive que le bourreau fasse mal son travail, lorsqu'il rate son "chefd'œuvre" de décapitation par exemple, la foule ne garde plus sa place de simple spectateur. Devenant parfois incontrôlable elle se range, soit du côté du condamné qu'elle peut tenter de libérer, soit du côté du pouvoir en cherchant à le mettre à mort. De toutes les façons, elle s'attaque en premier lieu au bourreau, transformé, respectivement, soit en agent de violences illicites, soit en victime qui mérite de l'être (Spierenburg 1984, Chapitre 2). Ainsi, l'équilibre délicat entre les quatre termes en présence -- autorités, condamné, public et bourreau -- est rompu, et tout l'édifice, basculant hors du cadre rituel, s'effondre, en quelque sorte sur le dos du bourreau, en une simple relation duelle entre agresseur et agressé.

L'imposition de la douleur dans ces supplices européens répond à l'évidence à un schéma asymétrique, la douleur elle-même pouvant être qualifiée de négative et infamante. Tournons nous maintenant vers un autre cas où, au contraire, la souffrance est infligée dans le cadre d'une réciprocité présumée et vécue comme positive et honorable.

\footnotetext{
${ }^{5}$ Les diverses explications avancées pour rendre compte de cette ambivalence (cf. par exemple Spierenburg 1984), dépendent soit d'un symbolisme exogène au rituel d'exécution, soit d'une psychologie (de masse) ad hoc, et demeurent, de ce point de vue, insuffisantes.
} 
A une époque peu éloignée dans le temps de celle qu'on vient d'évoquer, au cours des XVIe et XVIIe siècles, mais de l'autre côté de l'Atlantique, les Indiens du groupe linguistique Iroquois de l'Amérique du Nord pratiquaient régulièrement la torture sévère des captifs de guerre. D'après de nombreux témoignages, notamment ceux des missionnaires jésuites (cf. les sources citées dans Knowles 1940 ; Tooker 1987), le scénario était partout plus ou moins le même :

Une fois capturé, le prisonnier, habillé de façon séante, était accompagné de village en village avant d'arriver à celui où il devait être livré. A chaque arrêt, on lui apportait de la nourriture et on lui ordonnait de chanter. Arrivé à sa destination, le captif était remis à une famille ayant perdu un de ses membres et qui pouvait donc l'adopter. Toutefois, il arrivait souvent que son corps soit déjà trop endommagé -- on lui infligeait parfois des violences en route -- et on décidait plutôt de sa mise à mort. A partir de ce moment il était traité avec grand respect et sollicitude.

Le supplice du prisonnier se passait en deux temps. En compagnie des seuls hommes, il était torturé la nuit durant dans la maison des conseils de guerre. Ayant allumé une rangée de feux le long de la maison, les hommes obligeaient le captif, les mains liées, à en faire plusieurs fois le tour -- d'abord en chantant et en dansant puis simplement en courant -- pendant que chacun avec son tison le brûlait au passage. Étouffant ses cris sous des hurlements, les jeunes guerriers l'arrêtaient de temps en temps pour le faire s'allonger sur des braises ardentes, pour lui briser les os des mains, lui percer les oreilles avec des baguettes, etc. Lorsque le prisonnier s'effondrait, on lui donnait à manger et à boire et on le laissait se reposer avant de recommencer les tortures. A ces moments-là, la cruauté des jeunes guerriers s'accompagnait de propos d'une tendresse ironique : l'un le brûle sévèrement en prétextant qu'il doit aider son "oncle" qui a froid à se réchauffer, un autre lui offre comme cadeau une hache chauffée au rouge qu'il lui applique sur les pieds, un troisième, soi-disant pour le rendre beau, l'habille avec de vieux vêtements auxquels il met ensuite le feu, etc. "Eh bien mon oncle est-ce assez?" lui demandent-ils souvent, et lorsqu'il répond "Oui mon neveu, c'est assez, c'est assez", ils répliquent "Non, ce n'est pas assez" et continuent à le brûler. A chaque moment d'arrêt de ce long processus, on exigeait que le captif entonne son chant de guerre dans lequel il détaillait le courage des siens et la vengeance qui ne manquerait pas de s'abattre sur ceux qui le malmènent. Et lorsqu'il se montrait réticent à continuer, on lui rappelait la façon qu'avaient lui et les siens de traiter leurs captifs : "N'étais-tu pas bien cruel à l'endroit des prisonniers ? Dis-nous un peu, n'as tu pas pris plaisir à les brûler ? Tu ne pensais pas qu'on devait te traiter de la sorte, mais peut-être pensais-tu avoir tué tous les Hurons ?"

A l'aube, le captif était conduit dehors sur une estrade aménagée autour d'un arbre auquel il était attaché de telle sorte qu'il pouvait tourner autour. Devant le village assemblé, plusieurs hommes montés avec lui se mettaient alors à le brûler avec une intensité redoublée partout sur le corps. Lorsque le captif tombait sans pouvoir être réanimé, de peur qu'il ne meurt sous la torture, on le tuait rapidement en lui tranchant la gorge. Si le prisonnier avait été particulièrement brave avant de mourir, on faisait ensuite un festin de son corps, les guerriers mangeant son cœur afin de devenir courageux.

Ici, comme dans les supplices européens, la douleur infligée instaure une distance et une différence indéniable entre ceux qui imposent la souffrance et celui qui la subit. 
Toutefois, on constate en même temps une série d'éléments allant dans un sens inverse, c'est-à-dire dans celui d'un rapprochement entre le captif et ses tortionnaires. Le prisonnier, qu'on désigne comme "neveu" ou "oncle" selon l'âge relatif des interlocuteurs, est d'emblée considéré comme un parent ou un affin virtuel (toute torture est une adoption manquée, cf. Simonis 1977:117). Il est traité avec déférence et bienveillance ; même la consommation de sa chair serait un signe supplémentaire d'estime. C'est également dans cette optique qu'il faut comprendre l'insistance avec laquelle les tortionnaires font chanter leur victime : "On attendait de la victime qu'elle chante et danse à tout moment. Il le faisait lorsqu'un festin d'adieu était donné avant que débute le supplice [...] et dans la cabane au cours de la nuit de torture. Les chansons étaient surtout des vantardises qui soulignaient l'absence de peur de la part du chanteur." (Knowles 1940:187-8) Comme le remarque cet auteur (ibid.), il y a peu d'indications qui laissent penser que les tortionnaires essayaient de "casser" la volonté du prisonnier. Bien au contraire, en exigeant que celui-ci continue à chanter son chant de guerre, ils l'amènent à correspondre à l'image du guerrier tel qu'ils se voient eux-mêmes -- ou plutôt, comme le suggère l'ingestion finale du captif qui s'est ainsi montré courageux, tels qu'ils voudraient être. L'attitude attendue d'un guerrier aux mains de l'ennemi semble bien résumée dans les propos d'un homme (Seneca) qui, après avoir été torturé, s'est échappé et a pu rapporter son aventure aux siens (ibid. 188) : "Il s'est vanté de ne ressentir aucune douleur, il s'est glorifié de ses accomplissements guerriers, il leur a dit ce que les Seneca leur feraient pour se venger et puis il a chanté sa chanson d'adieu."

Si la victime est rendue ainsi semblable à ses tortionnaires, réciproquement, ces derniers sont potentiellement identifiés au guerrier qu'ils détiennent. A travers les menaces de vengeance que profère le prisonnier et l'évocation de situations où lui et les siens ont agi en tortionnaires, se trouvent représentés des supplices passés et futurs dans lesquels les positions respectives des protagonistes sont interverties, chacun occupant la place de l'autre. La relation entre la victime et ses tortionnaires se trouve donc comme doublée d'une inversion virtuelle dans le cadre de laquelle la férocité des supplices pratiqués par les uns témoigne de la cruauté des autres, et l'héroïsme que manifeste, à l'aide des tortionnaires, le prisonnier, devient un indice de leur propre courage. Cette inscription rituelle du supplice dans le contexte de son propre renversement fait de cette relation on ne peut plus asymétrique le fondement d'une réciprocité dynamique, à savoir celle de la vengeance généralisée.

L'identification entre le meurtrier et sa victime qui est au fondement du système relationnel mis en place au cours de ce supplice se retrouve dans d'autres parties du monde (cf. par exemple Cartry et Détienne 1996; Harrison 1993). Dans les sociétés voltaïques de l'Afrique de l'Ouest par exemple, le traitement rituel auquel est soumis le meurtrier serait fondé sur l'idée que la "vie de la victime" s'est "insinuée dans le corps du meurtrier" (Cartry 1996:285), qui devient ainsi "la proie de sa victime" (ibid. 296). "Dès l'instant où le meurtre a été accompli, le corps de l'homicide est littéralement assailli par sa victime qui le 'tire dans tous les sens', le 'frappe', 'l'empêche de dormir', le 'rend fou' et, à court terme, finit par le 'tuer', si le meurtrier ne se soumet pas aux rites de l'homicide." (Liberski-Bagnoud 1996:241) De façon similaire, dans de nombreuses sociétés amazoniennes, à la suite d'un homicide, souvent accompli dans le cadre d'un cannibalisme réel ou symbolique, le meurtrier "se trouve assujetti aux influences corporelles de sa victime, ou aux pouvoirs spirituels de celle-ci en tant qu'inhérents à certaines substances vitales" (Viveiros de Castro 1996:93, cf. également Taylor 1994; Menget 1996). Par exemple, les Arawete du Brésil disent d'un homme qui a tué qu'il "meurt" : il sent ses os se ramollir, il entend les vautours qui se rassemblent autour de son corps mort, etc. Tombant dans un état de stupeur, il reste immobile sans manger pendant plusieurs jours, ne faisant que vomir le sang de son ennemi qui lui remplirait le ventre. Il est habité par l'esprit de sa victime qui lui fait ensuite exécuter une danse de guerre, lui soufflant 
les paroles d'un chant où le sujet d'énonciation est situé dans un mouvement de réverbération constante entre le meurtrier et sa victime (Viveiros de Castro 1996:81-6). Signalons enfin qu'on trouve dans un dialogue rituel entre le tueur et sa victime préalable à un festin cannibale chez les Tupinamba du XVIe siècle une recontextualisation du meurtre qui est assez semblable à celle signalée chez les Iroquois : le tueur exhorte la victime à mourir de façon courageuse et la victime demande qu'on la tue afin qu'elle soit vengée, disant même que les siens se sont déjà vengés sur ceux qui sont sur le point de l'abattre (sources citées dans Viveiros de Castro 1992:291).

Toutefois, dans le cas Iroquois l'opération par laquelle s'instaure cette relation rituelle quelque peu singulière entre l'agresseur et l'agressé n'est pas le meurtre mais la torture ; l'expérience charnière n'est pas tant la mise à mort que la souffrance infligée. Cette différence semble comporter une certaine transformation du schéma habituellement rencontré. Les cas précédemment cités décrivent une identification de l'agresseur à l'agressé qui se rapporte à un processus intervenant à la suite de l'homicide et/ou du festin cannibale : le meurtrier est assujetti par sa victime au moyen d'une incorporation ou intériorisation dangereuse. En revanche, dans le cas Iroquois, on assiste à une identification de l'agressé aux agresseurs qui se rapporte à un processus qui précède la mort et la consommation du prisonnier (lesquelles n'entraîneraient apparemment pas de dangers mystiques particuliers [Tooker 1987:139]) : le captif est assujetti par ses tortionnaires qui s'attaquant à la surface extérieur de son corps (en faisant chanter leur victime).

Ce premier niveau de divergence en recouvre un deuxième. Se référant aux Arawete et à d'autres sociétés amazoniennes, Viveiros de Castro suggère que le meurtre intertribale et les pratiques qui lui sont liées, comme le cannibalisme et le traitement des trophées, comporterait avant tout une "assomption du point de vue de l'ennemi" (Viveiros de Castro 1996:99). Ainsi, "plutôt que des substances ou principes plus ou moins réifiés que chaque société (ou chaque ethnographe) choisit comme substrat et objet des processus d'assimilation de l'ennemi [...], [ce] qu'on assimile de la victime, ce sont les signes de son altérité, et ce qu'on vise c'est cette altérité comme point de vue ou perspective sur Soi" (1996:98; cf. aussi Erikson 1986). Adoptant une perspective similaire, on remarquera alors que si les tortionnaires iroquois peuvent eux aussi être associés à l'ennemi, c'est parce qu'ils imposent leur propre point de vue sur le captif, le contraignant à s'adapter à l'image qu'ils ont d'eux-mêmes. Dans le premier cas, c'est la perspective de l'agressé qui est privilégiée, dans le second, celle de l'agresseur. La torture iroquoise des captifs n'est pas, comme le meurtre tupinamba, "un mécanisme symbolique de devenir-l'Autre" (Viveiros de Castro 1992:270), mais au contraire un dispositif symbolique pour ramener l'autre à Soi. Il est vrai que dans le cadre d'une réciprocité supposée -- un régime de vengeance généralisée et diffuse -- ces différentes démarches aboutissent l'une comme l'autre à un échange de points de vue. Elles sous-tendent donc toutes deux la mise en place d'une société qui "doit être comprise comme incluant ses ennemies, [qui] s'est constituée au travers de sa relation avec d'autres, en un régime d'hétéronomie généralisée" (ibid. 283). Cependant, le type de "socialité" (Strathern 1988) en jeu, et plus précisément l'orientation de cette relation avec l'Autre, n'est pas le même. Dans le cas amazonien il s'agit de se poser (en tant qu'ennemi) parmi d'Autres, tandis que dans le cas iroquois, il s'agit d'assimiler des Autres (en tant qu'ennemis) à soi. Si le premier peut être qualifié de "prédation ontologique" (Viveiros de Castro 1996:98), le second fait figure de processus annexionniste. Ainsi, on ne s'étonnera guère d'apprendre que l'important développement de la torture chez les Iroquois (ainsi que la formation chez eux de confédérations ou ligues) semble avoir été une évolution tardive en réponse à un accroissement des rapports guerriers avec d'autres populations de la région. Cette pratique, qui aurait entraîné "une expansion de la terreur des Iroquois, utilisée et accentuée par eux afin d'obtenir une soumission de leurs ennemis" 
(Knowles 1940:219-20), aurait en même temps provoqué une conversion mimétique de ces derniers : de nombreux groupes indiens ont eux-mêmes adopté la torture des prisonniers à titre de représailles à la suite de contacts avec les Iroquois (ibid. 190-1) ${ }^{6}$.

La douleur infligée, en ce qu'elle associe de façon particulièrement manifeste affect et connexion interpersonnelle, peut représenter un opérateur important dans l'action rituelle, laquelle est fondée sur une mise en scène de relations. Si on peut douter de la vérité d'une douleur simplement ressentie par quelqu'un d'autre (cf. Scarry 1985), la réalité d'une douleur expressément infligée devient pratiquement indéniable, non seulement pour ceux qui assistent à la scène concernée mais aussi pour ceux qui l'anticipent, pour ceux qui en ont entendu parler, etc. Une souffrance imposée est en effet aisément investie du vécu et de l'imagination de chacun. L'épaisseur intentionnelle dont bénéficie la douleur infligée la dote en même temps d'une réalité d'autant plus partageable. De plus, cette douleur incontestable, précisément parce qu'elle est infligée, ne peut pas être dissociée du contexte relationnel dans lequel elle se réalise. Ailleurs, j'ai essayé de montrer que, dans le cadre d'activités rituelles, ce contexte est organisé d'une façon distinctive, à savoir par une condensation conventionnelle de modalités de relation contradictoires (cf. Houseman et Severi 1994). Nous observons une dynamique similaire dans les cas qu'on vient d'examiner. Ainsi, dans les supplices publics en Europe comme dans la torture des captifs chez les Iroquois, la douleur infligée instaure entre les agresseurs et le supplicié une rupture existentielle radicale, une démonstration flagrante d'altérité. Mais en même temps, dans ces deux cas, cette relation de différence et de distance est associée à d'autres relations, faisant intervenir d'autres termes, qui établissent au contraire un rapprochement entre ces antagonistes. Dans le cas européen, ce rapprochement se fait sur un mode asymétrique, à travers la médiation du public spectateur (et de sa "réplique" en positif incarnée par le bourreau), virtuellement identifié à la fois aux autorités et au condamné. Dans le cas iroquois, le rapprochement se fait sur un mode symétrique, sous la forme d'un renversement virtuel du rapport entre tortionnaires et victime (faisant intervenir d'autres occasions de torture passées et futures) où chacun prend la place de l'autre. Le réseau de connexions actualisé dans chacun de ces deux usages cérémoniels de la souffrance définit un type de relation spécifique, particulièrement complexe : une relation rituelle. Celle-ci, mise en place, dans un cas entre les représentants du pouvoir et les sujets citoyens du Haut Moyen Age européen, et dans l'autre entre les adversaires guerriers de populations iroquoises des XVIe et XVIIe siècles, est à la fois structurée par l'action cérémonielle et imbue de l'immédiateté d'une expérience corporelle irrécusable.

\section{Conclusion}

Il est évident que je n'ai pas fait le tour ici de l'ensemble des types de phénomènes centrés sur la douleur, ni des différents types de situations comportant une douleur infligée, ni même des différentes façons dont la douleur infligée peut intervenir dans le cadre de l'action rituelle. Les rites thérapeutiques par exemple représentent à eux seuls un champ d'investigation énorme. La douleur imposée à soi-même mais impliquant un tiers observateur, notamment dans le cadre d'épreuves sportives (cf. Le Breton 1995:204-8), serait, comme la douleur chronique, fondée sur une configuration relationnelle de un et demi. Toutefois, sa

\footnotetext{
${ }^{6}$ De ce point de vue, les Iroquois et les Européens ont occupé vis-à-vis des autres populations indiennes de l'Est de l'Amérique du Nord une position tout à fait similaire : "Il est établi avec certitude que les tribus de la côte atlantique n'ont pratiqué la torture des captifs qu'après une longue période de contact avec les blancs, et lorsque cette pratique a enfin fait son apparition, c'était en représailles contre un traitement similaire aux mains des blancs ou des Iroquois." (Knowles 1940:202-3)
} 
prise en considération exigerait l'ajout d'une discrimination supplémentaire entre des cas où la personne qui subit la douleur occupe un rôle passif et ceux où elle intervient de façon plus active. C'est également sur ce versant actif ou "volontariste" qu'il convient de situer -probablement au niveau d'une configuration relationnelle de deux et demi -- les deux grands absents de cette discussion sur la douleur infligée que sont d'un côté les rapports sadomasochistes et de l'autre le rapport entre soignant et patient dans le cadre de soins douloureux (cf. Peter 1993 et Marta 1997 pour deux perspectives différentes). Notons enfin que le champ de la douleur infligée qu'on a voulu circonscrire ici peut être pensé comme étant celui de la violence, rapprochement qui aurait le mérite de suggérer la définition suivante : est violence tout cas de douleur ressentie comme infligée.

L'ambition des spéculations qui précèdent n'était donc pas de proposer un cadre théorique achevé mais de fixer quelques repères pour une éventuelle étude comparative proprement anthropologique des phénomènes de douleur. Cette étude doit porter moins sur la douleur en tant qu'expérience privée que sur les conditions sociales de cette expérience, c'està-dire sur les relations qu'entretiennent les divers agents qui y sont impliqués (qu'ils soient réels ou imaginaires, visibles ou invisibles). Une telle optique requiert, selon moi, une certaine réorientation quant à l'objet même de la recherche. Elle exige notamment qu'on envisage comme cas le plus élémentaire de la douleur -- où ses particularités seraient le plus explicitement réalisées -- non pas, comme en médecine, la douleur simplement ressentie, mais la douleur infligée.

Dans son remarquable ouvrage The Body in Pain, Elaine Scarry (1985) décrit de façon éloquente la capacité qu'a la douleur de soustraire du sens à l'expérience, de "défaire le monde" (world unmaking). Cet anéantissement du sens serait, selon elle, le propre de la souffrance. J'ai proposé ici d'analyser divers phénomènes organisés autour de la douleur infligée en considérant la structure relationnelle qui sous-tend la souffrance qui y intervient. Cette démarche a fait ressortir, en autres choses, un certain ordonnancement possible de ces phénomènes. La douleur chronique, caractérisée par une vaine tentative de dédoublement au sein même de la personne souffrante, répondrait à une configuration relationnelle que j'ai qualifiée de "un et demi". La torture politique, qui se distinguerait par la présupposition d'une relation entre deux relations duelles, serait fondée sur une configuration de "deux et demi". Enfin, l'usage rituel de la douleur relèverait d'une configuration comportant l'interdépendance systématique d'au moins trois relations. J'ai été ainsi amené à tempérer la position de Elaine Scarry : plus la configuration relationnelle en présence est complexe, c'est-à-dire plus elle intègre de relations entre des agents qui se représentent les représentations qu'auraient les autres, plus la douleur concernée contribuerait à la "construction d'un monde" (world making).

Ce potentiel créateur de la douleur - en d'autres termes, son aptitude à participer à la mise en place et à la transmission des éléments d'une tradition (un "monde") - est directement lié à l'importance des contraintes qui président à son imposition. Dans la douleur chronique, la supposition d'une relation avec un protagoniste responsable de la douleur engage essentiellement le malade concerné. Celui-ci bénéficie donc d'une très grande liberté quant à la façon dont il caractérisera son malheur et quant à la manière dont il envisagera ses rapports avec ce ou celui qu'il suppose être à l'origine de sa douleur. C'est en modifiant constamment la nature de cette relation supposée, notamment au moyen de divers types de dédoublement (moi/mon corps, moi inconscient/moi conscient, moi privé/moi public, etc.) que le sujet tente de faire face à sa souffrance. Dans la torture politique, la douleur est infligée en fonction de l'association de deux relations duelles : celle obéissant à une logique de l'interrogation entre le tortionnaire et sa victime, et celle obéissant à une logique de la terreur entre le tortionnaire 
et des personnes tierces. En comparaison avec la douleur chronique, où il n'y a pas à proprement parler de relation en présence, les interactions, paroles, etc. intervenant dans l'imposition de la douleur entrent donc nécessairement plus dans un ensemble de contraintes. Toutefois, dans la mesure où la relation supplémentaire que présupposent ces deux premières relations et qui les relie entre elles, à savoir celle entre la victime et les personnes tierces, n'est pas actualisée mais simplement supposée, le tortionnaire bénéficie d'une marge de manœuvre considérable. C'est par une exploitation stratégique de cette marge qu'il pourra jouer l'une de ces relations contre l'autre : ce qui ne peut se justifier par rapport à la démarche interrogatrice s'expliquerait aisément par les exigences de la terreur et vice-versa. Enfin, dans l'activité rituelle, la douleur est infligée dans le cadre de l'interconnexion systématique d'une pluralité de relations actualisées. Il y a donc là un maximum de contraintes sur les actions et sur les paroles associées à l'imposition de la souffrance. Dans ces conditions, la mise en place de dispositifs interactifs et discursifs particulièrement stables est fortement favorisée.

En passant de la douleur chronique à la torture politique et de celle-ci à l'usage cérémoniel de la souffrance, on a pu constater une augmentation progressive des contraintes portant sur les actions, les paroles et les représentations intervenant dans l'imposition de la douleur. Cette augmentation des contraintes sous-tend un accroissement du potentiel créatif de ces diverses pratiques, c'est-à-dire de leur aptitude à contribuer à l'émergence d'un monde partagé -- d'une tradition. Or, cette progression dépend en premier lieu, non pas de la présence de scénarios préexistants de plus en plus compliqués ou de plus en plus clairement définis, mais de la mise en scène au cours de ces diverses activités de formes relationnelles de plus en plus complexes. Ce sont ces formes relationnelles qui fournissent aux acteurs l'échafaudage nécessaire à l'élaboration de scénarios éventuels. 


\section{Bibliographie}

Alleg, $\mathrm{H}$.

1961 La question. Paris, Éditions de Minuit.

Asad, T.

1993 "Pain and Truth in Medieval Christian Ritual", in T. Asad Genealogies of Rzeligion. Baltimore, The John Hopkins University Press.

Baszanger, I.

1995 "Théorie, techniques et prise en charge : le cas de deux centres de traitement de la douleur", Techniques et culture 25-26:263-284.

Bee, $M$.

1983 "Le spectacle de l'exécution sous l'Ancien Régime", Annales Economies, Sociétés Civilisations 38 (4) : 843-862.

Bernard, M.

1982 "Les paradoxes de la douleur", Esprit 2.

Bernand, C. et S. Fisher

1974 "Les mots de la tribu", L'Homme 14 (2) : 137-138.

Buytendjik, F.J.J.

1951 De la douleur. Paris, P.U.F.

1978 "La douleur vécue" in Encyclopaedia Universalis. Paris, Encyclopaedia Universalis France, pp.782785.

Cartry, M.

1996 "La dette sacrificielle du meurtrier d'après quelques récits d'administrateurs-ethnologues (Afrique de l'Oeust)", Systèmes de Pensée de Afrique Noire 14 : 77-104 (Destins de meurtriers, Textes réunis par M. Cartry et M. Detienne).

Cartry, M. et M. Detienne (éds.)

1996 Systèmes de Pensée de Afrique Noire 14 : 77-104 (Destins de meurtriers), Paris.

Clastres, P.

1973 "De la torture dans les sociétés primitives", L'Homme 13 (3) : 114-120.

Cohen, E.

1990 "'To Die a Criminal for the Public Good': The Execution Ritual in Late Medieval Paris", in B.S. Bachrach et D. Nicholas (éds.) Law, Custom and the Social Fabric in Medieval Europe. Kalamazoo, Cohen, $\mathrm{M}$

Medieval Institute Publications, Western Michigan University

1964 "Establishment of Identity in a Social Nexus", American Anthropologist 66 : 529-552.

Csordas, T. et J. Clark

1992 "Ends of the Line: Diversity among Chronic Pain Centers", Social Science and Medicine 34 (4) : 383 394.

Delarue, J.

1979 Le métier de bourreau. Du Moyen-Age à aujourd'hui. Paris, Fayard.

Delvecchio Good, M.-J., P.E. Brodwin, B.J. Good et A. Kleinman (éds.)

1992 Pain as Human Experience: An Anthropological Perspective. Berkeley, University of California Press. Droogers, A.

1980 The Dangerous Journey. Symbolic Aspects of Boy's Initiation among the Wagenia of Kisangani, Zaire.

Erikson, $\mathrm{Ph}$ La Haye, Mouton.

1986 "Altérité, tatouage et anthropophagie chez les Pano : la belliqueuse quête de soi", Journal de la Société des Américanistes de Paris 72 : 185-210.

Forrest, D

1996 "The Methods of Torture and Its Effects", in D. Forrest (éd.) A Glimpse of Hell. Reports on Tioture Worldwide. Londres, Amnestry International / Cassell.

Foucault, M.

1975 Surveiller et punir. Naissance de la prison. Paris, Gallimard.

Good, B.J.

1992 "A Body in Pain -- The Making of a World of Chronic Pain", in M.-J. DelVecchio Good, P.E. Brodwin, B.J. Good and A. Kleinman (éds.) Pain as Human Experience: An Anthropological Perspective. Berkeley: University of California Press.

Gonthier, N.

1992 Cris de haine et rites d'unité. La violence dans les villes, XIII - XIV siècle. Bruxelles, Brepols. 
Harrison, S.

1993 The Mask of War: Violence, Ritual and the Self in Melanesia. Manchester, Manchester University Press.

Hilbert, R.A.

1984 "The Acultural Dimension of Chronic Pain: Flawed Reality Construction and the Problem of Measuring. Social Problems 31 (4) : 365-378.

Houseman, M.

1986 "Le mal pour le mâle : un bien initiatique", in J. Hainard et R. Kaehr (eds.), Le Mal et la Douleur. pp. 89-99. Neuchâtel, Musée d'ethnographie.

1993 "The Interactive Basis of Ritual Effectiveness in a Male Initiation Rite", in P. Boyer (ed.) Cognitive Aspects of Religious Behaviour. Cambridge, Cambridge University Press.

Houseman, M. and C. Severi

1994 Le naven ou le donner à voir : vers une théorie de l'action rituelle. Paris: Editions MSH / CNRS.

Jackson, J.

1994 "Chronic Pain and the Tension between the Body as Subject and Object", in T.J. Csordas (ed.) Embodiment and Experience. The Existential Ground of Culture and Self. Cambridge: Cambridge University Press.

Jempson, M.

1996 "Torture Worldwide", in D. Forrest (éd.) A Glimpse of Hell. Reports on Torture Worldwide. Londres, Amnestry International / Cassell.

Knowles, N.

1940 "The Torture of Captives by Indians of Eastern North America", Proceedings of the American Philosophical Society 82 (2) : 151-225.

Le Breton, D.

1995 Anthropologie de la douleur. Paris, Editions Métailié.

Liberski-Bagnoud, D.

1996 "La lame du couteau et la mort amère (Kasena, Burkina Faso)", Systèmes de Pensée de Afrique Noire 14 : 77-104 (Destins de meurtriers, Textes réunis par M. Cartry et M. Detienne).

Margarido, A. et M. Panoff

1974 "De l'ethnologie comme torture des faits?", L'Homme 14 (2) : 139-142.

Marr, D.

1982 Vision: A Computational Investigation into the Human Representation and

Processing of Visual Information. New York, Freeman.

Marta,

1997 Sauver sa peau.

Melzack, R. et P. Wall

1983 The Challenge of Pain. New York, Basic Books.

Menget, $\mathrm{P}$.

1996 "De l'usage des trophées (Amérique du sud)", Systèmes de Pensée de Afrique Noire 14 : 77-104 Morris, D.M.

(Destins de meurtriers, Textes réunis par M. Cartry et M. Detienne).

1991 The Culture of Pain. Berkeley, University of California Press.

Muchembled, R.

1992 Le temps des supplices. De l'obéissance sous les rois absolus. XVe-XVIIIe siècle. Paris, Armand Colin. Orwell, G.

1950 1984. Paris, Editions Gallimard.

Peter, J.P.

1993 "Trois 'Propos sur la douleur'. Observations sur les attitudes de la médecine prémoderne envers la Peters, E. douleur", in J.-P. Peter (ed.) De la douleur. Paris: Quai Voltaire.

1985 Torture. Oxford : Basil Blackwell.

Rétat, P. (sous la direction de)

1979 L'attentat de Damiens. Discours dur l'événement au XVIIIe siècle (Centre d'Etudes du XVIIIe Siècle, Université Lyon II). Paris / Lyon, Editions du CNRS / Presses Universitaires de Lyon.

Rey, R.

1993 Histoire de la douleur. Paris, La Découverte.

Scarry, E.

1985 The Body in Pain. The Making and the Unlaking of the World. Oxford: Oxford University Press.

Shue, $\mathrm{H}$. 
1978 "Torture", Philosophy and Public Affairs 7 (2) : 124-143.

Simonis, Y.

1977 "Le canibalisme des Iroquois : comportement social, environnements, structures de l'esprit", Anthropologie et sociétés. 1 (2) : 107-122.

Spieirenburg, $\mathrm{P}$.

1984 The Spectacle of Suffering. Executions and the evolution of repression: from a preindustrial metropolis to the European experience. Cambridge: Cambridge University Press.

Strathern, M.

1988 The Gender of the Gift. problems with Women and Problems with Society in Melanesia. Berkely, University of California Press.

Taylor, A.-Ch.

1993 "Le bons ennemis et les mauvais parents : le traitement symbolique de l'alliance dans les rituels de chasse aux têtes des Jivaros de l'Equateur", in E. Copet et F. Héritier-Augé (éds.) Les complexités de l'alliance, IV. Economie, politique et fondements symboliques de l'alliance. Paris, Archives Contemporaines.

Tooker, E.

1987 Ethnographie des Hurons, 1615 - 1649. Montréal, Recherches amérindiennes au Québec [1964].

Viveiros de Castro, E.

1992 From the Enemy's Point of View. Humanity and Divinity in an Amazonian Society. Chicago, University of Chicago Press.

1996 "Le meurtrier et son double (Araweté, Amazonie)", Systèmes de Pensée de Afrique Noire 14 : 77-104 (Destins de meurtriers, Textes réunis par M. Cartry et M. Detienne).

Violon, A.

1992 La douleur rebelle. Paris, Desclée de Brouwer.

Woolf, V.

1981 The Movement and Other Essays. London: Hoggarth Press [1947].

Zempleni, A.

1976 "La chaîne du secret", Nouvelle Revue de Psychanalyse 14 : 413-424 (Du secret).

1993 "L'invisible et le dissimulé. Du statut religieux des entités initiatiques", Gradhiva 14 : 3-14. 